\title{
Analysis of Host Species Specificity of Magnaporthe grisea Toward Foxtail Millet Using a Genetic Cross Between Isolates from Wheat and Foxtail Millet
}

\author{
J. Murakami, R. Tomita, T. Kataoka, H. Nakayashiki, Y. Tosa, and S. Mayama
}

Faculty of Agriculture, Kobe University, Nada, Kobe 657-8501, Japan.

Accepted for publication 8 August 2002.

\begin{abstract}
Murakami, J., Tomita, R., Kataoka, T., Nakayashiki, H., Tosa, Y., and Mayama, S. 2003. Analysis of host species specificity of Magnaporthe grisea toward foxtail millet using a genetic cross between isolates from wheat and foxtail millet. Phytopathology 93:42-45.

Host species specificity of Magnaporthe grisea toward foxtail millet was analyzed using $\mathrm{F}_{1}$ cultures derived from a cross between a Triticum isolate (pathogenic on wheat) and a Setaria isolate (pathogenic on foxtail

Ki-awa, two loci were involved and one of them was Pfml. The other locus was designated as $P f m 2$. Interestingly, $P f m l$ was not involved in the pathogenic specificity on cv. Kariwano-zairai. These results suggest that there is no "master gene" that determines the pathogenic specificity on all foxtail millet cultivars and that the species specificity of $M$. grisea toward foxtail millet is governed by cultivar-dependent genetic mechanisms that are similar to gene-for-gene interactions controlling racecultivar specificity.
\end{abstract} millet). On foxtail millet cvs. Beni-awa and Oke-awa, avirulent and virulent cultures segregated in a 1:1 ratio, suggesting that a single locus is involved in the specificity. This locus was designated as Pfml. On cv.
Additional keywords: Pyricularia, resistance.
The basis of host-parasite specificity between plants and microorganisms has been discussed by many phytopathologists. Day (1) distinguished two types of host-parasite interaction: one determines whether or not a plant is a host for a parasite, and the other determines the gene-for-gene specificity. Heath (3) termed the specificity determining host species range as plant species specificity, and the specificity determining cultivar range within a given host species as cultivar specificity. In the former, compatibility is specific, and the resistance involved (nonhost resistance) is genetically complex. In the latter, incompatibility is specific, and the resistance involved (cultivar resistance) is controlled by gene-for-gene interactions. However, data have been reported that may be interpreted as suggesting that gene-for-gene interactions underlie the plant species specificity (4).

Magnaporthe grisea (Hebert) Barr, the causal agent of blast disease, includes several subgroups that are pathogenic to a restricted range of plant species, i.e., Oryza pathotype pathogenic to rice (Oryza sativa L.), Setaria pathotype pathogenic to foxtail millet (Setaria italica Beauv.), Panicum pathotype pathogenic to common millet (Panicum miliaceum L.), Eleusine pathotype pathogenic to finger millet (Eleusine coracana (L.) Gaertn.), and Digitaria pathotype pathogenic to crabgrass (Digitaria sanguinalis (L.) Scop.) (7). In the 1980s, a new subgroup (Triticum pathotype) of $M$. grisea arose on wheat (Triticum aestivum (L.) Thell.) in Brazil and has spread to other countries in South America $(18,19)$. This specificity between the pathotypes and gramineous plant species appears to be plant species specificity according to the traditional usage. However, there are some reports suggesting that the plant species specificity of $M$. grisea is governed by a rather simple mechanism. Yaegashi (20) crossed Eleusine isolates

Corresponding author: Y. Tosa; E-mail address: tosayuki@kobe-u.ac.jp

Publication no. P-2002-1104-01R

(C) 2003 The American Phytopathological Society with weeping lovegrass isolates and suggested that their pathogenicity to finger millet or weeping lovegrass is conditioned by a single gene. Kang et al. (5) and Sweigard et al. (10) found two unlinked major genes, $P W L 1$ and $P W L 2$, which control species specificity to weeping lovegrass, and showed that they have characteristics similar to avirulence genes that condition cultivar specificity.

To elucidate the genetic mechanisms controlling the species specificity in $M$. grisea, we produced two hybrid populations. One was derived from a cross between a Triticum isolate and an Avena isolate. Using this population, Takabayashi et al. (11) showed that a gene-for-gene interaction underlies the avirulence of the Avena isolate on wheat. The other hybrid population was derived from a cross between a Triticum isolate and a Setaria isolate. Using this population, Murakami et al. (9) showed that the specific parasitism of the parental isolates on wheat is conditioned by two loci, $P w t 1$ and Pwt2. Although this result suggested that the species specificity on wheat is controlled by a rather simple genetic mechanism, it was not clear whether these genes conditioned virulence or avirulence. The objective of this study was to determine genetic mechanisms of their specific parasitism on the other host species, foxtail millet. The results suggested that the specificity toward foxtail millet is governed by cultivar-dependent genetic mechanisms that are similar to gene-for-gene interactions controlling race-cultivar specificity.

\section{MATERIALS AND METHODS}

Fungal strains. Parental strains were two field isolates of $M$. grisea, Br48 and GFSI1-7-2. Br48 was isolated from wheat in Brazil, whereas GFSI1-7-2 was isolated from foxtail millet in Japan. They were crossed on oatmeal-agar media and a total of 80 $\mathrm{F}_{1}$ cultures were obtained from 10 mature asci containing 8 ascospores (9). Preliminary cytological observation revealed that 11 of the 80 cultures were deficient mutants that lost the basic capacity of penetration. These mutants were excluded from further analysis. 
Plant materials. Setaria italica cv. Ki-awa (abbreviation, SI4), cv. Beni-awa (SI9), cv. Oke-awa (SI10), cv. Mosho-awa (SI12), and cv. Kariwano-zairai (SI17) were used as test plants for segregation analysis. Twelve seeds were sown in Sakata super-mix soil (Sakata, Yokohama, Japan) in a seedling case $(5.5 \times 15 \times 10 \mathrm{~cm})$ and grown at $26^{\circ} \mathrm{C}$ under natural light for 5 to 7 days.

Infection assays. $M$. grisea cultures were prepared as described previously (9). The conidia were suspended in water and adjusted to $1 \times 10^{5}$ conidia per $\mathrm{ml}$ with $0.01 \%$ Tween 20 . Twenty milliliters of the conidial suspension was sprayed on the adaxial surface of foxtail millet leaves with a glass sprayer connected to an air compressor. The inoculated seedlings were kept in a humid, dark box for $20 \mathrm{~h}$ at 20 to $22^{\circ} \mathrm{C}$. They were then transferred to a controlled-environment room with a 12 -h photoperiod of fluorescent lighting $(4,000 \mathrm{~lx})$ and incubated further at 20 to $22^{\circ} \mathrm{C}$. Five days after inoculation, symptoms were evaluated based on affected area on the leaves. The affected area was rated using six progressive grades from 0 to 5: 0 , no visible symptoms; 1 , diseased leaf area of up to $5 \%$ of the whole leaf, mainly composed of brown pinpoint spots; 2 , diseased leaf area of up to $10 \%$, mainly composed of small brown lesions; 3 , diseased leaf area of up to $50 \%$, mainly composed of green lesions with an intermediate size; 4 , diseased leaf area of up to $80 \%$, mainly composed of green large lesions; and 5, diseased leaf area of more than $80 \%$, almost completely shriveled. The mean disease severity on eight leaves was used as the virulence index (VI) of each culture. Isolates with VI less than 3 were considered avirulent, whereas those with VI greater than or equal to 3 were considered virulent. The inoculation assay was repeated at least five times with similar results.

\section{RESULTS}

Pathogenicity of the parental isolates toward foxtail millet cultivars. Primary leaves of foxtail millet cultivars were inoculated with the parental isolates. The Triticum isolate $\mathrm{Br} 48$ was avirulent on all the foxtail millet cultivars (Fig. 1; Table 1). In contrast, the Setaria isolate GFSI1-7-2 produced many watersoaked lesions on SI4, SI9, SI10, and SI17. They enlarged and coalesced with time and, finally, the whole leaf shriveled with its color remaining green by 5 days after inoculation (Fig. 1). Virulence indices of $\mathrm{Br} 48$ and GFSI1-7-2 on these four cultivars were approximately 1 and 5, respectively (Table 1). On SI12, however, both parental isolates produced no visible lesions (Table 1). This foxtail millet cultivar was assumed to carry a cultivar-specific resistance gene.

Segregation analysis of pathogenicity of $F_{1}$ cultures on foxtail millet cultivars. The foxtail millet cultivars were inoculated with the $69 \mathrm{~F}_{1}$ cultures derived from the $\mathrm{Br} 48 \times$ GFSI1-7- 2 cross.
On SI12 all the $F_{1}$ cultures were avirulent (Table 2). On the other cultivars, the $F_{1}$ cultures showed varying disease severity levels, with VI ranging from 0 to 5, but could be classified into avirulent or virulent. Figure 1 shows reactions of SI4, SI9, and SI17 to a set of tetrads $(1 \mathrm{~K} 1,1 \mathrm{~L} 1,1 \mathrm{M} 1$, and $1 \mathrm{~N} 1)$ as an example. Avirulent and virulent cultures segregated in 3:1, 1:1, and 3:1 ratios on SI4, SI9, and SI17, respectively. Interestingly, the patterns of reactions were various among the foxtail millet cultivars (Fig. 1).

The frequency distribution of VI in the $69 \mathrm{~F}_{1}$ cultures is summarized in Table 2. On SI9 and SI10, the segregation of avirulent and virulent cultures fitted a 1:1 ratio, suggesting that a single locus is involved in the specific pathogenicity of the parental isolates. The segregation patterns on these cultivars were identical; all $F_{1}$ cultures avirulent on SI9 were avirulent on SI10, whereas all $\mathrm{F}_{1}$ cultures virulent on SI9 were virulent on SI10 (Table 3). This result suggests that the specific pathogenicity to these cultivars is controlled by the same gene. This locus was designated as Pfml after pathogenicity on foxtail millet. On another cultivar SI4 avirulent and virulent cultures segregated in a 3:1 ratio (Table 2), suggesting that two unlinked loci are involved. The segregation pattern on SI4 was compared with that on SI9 to determine whether one of the two loci was Pfml. If one locus is identical to Pfml, $\mathrm{F}_{1}$ cultures that are avirulent on both SI9 and SI4, avirulent on SI9 only, avirulent on SI4 only, and virulent on both SI9 and SI4 should segregate in a 2:0:1:1 ratio. If both of the two loci are different from and independent of $P f m l$, they should segregate in a 3:1:3:1 ratio. The combined segregation on these cultivars fitted the former ratio (Table 3), suggesting that one of the two loci is Pfm1. The other locus was designated as Pfm2. On SI17 avirulent and virulent cultures also segregated in a 3:1 ratio although distribution of VI was somewhat continuous (Table 2). This result suggests that the pathogenicity to SI17 is conditioned by two loci. The combined segregation on SI9 and SI17 fitted a 3:1:3:1 ratio (Table 3), indicating that both of the two loci are different from

TABLE 1. Pathogenicity of parental isolates of Magnaporthe grisea to foxtail millet cultivars

\begin{tabular}{lccc}
\hline & & \multicolumn{2}{c}{ Virulence index $^{\mathrm{a}}$} \\
\cline { 3 - 4 } Cultivar & Abbreviation & GFSI1-7-2 & Br48 \\
\hline Ki-awa & SI4 & 4.6 & 1.0 \\
Beni-awa & SI9 & 4.8 & 1.1 \\
Oke-awa & SI10 & 4.8 & 1.2 \\
Mosho-awa & SI12 & 0.6 & 0.2 \\
Kariwano-zairai & SI17 & 4.5 & 1.0 \\
\hline
\end{tabular}

a The mean disease severity on eight leaves was used as the virulence index (VI) of each culture. Isolates with VI less than 3 were considered avirulent, whereas those with VI greater than or equal to 3 were considered virulent.

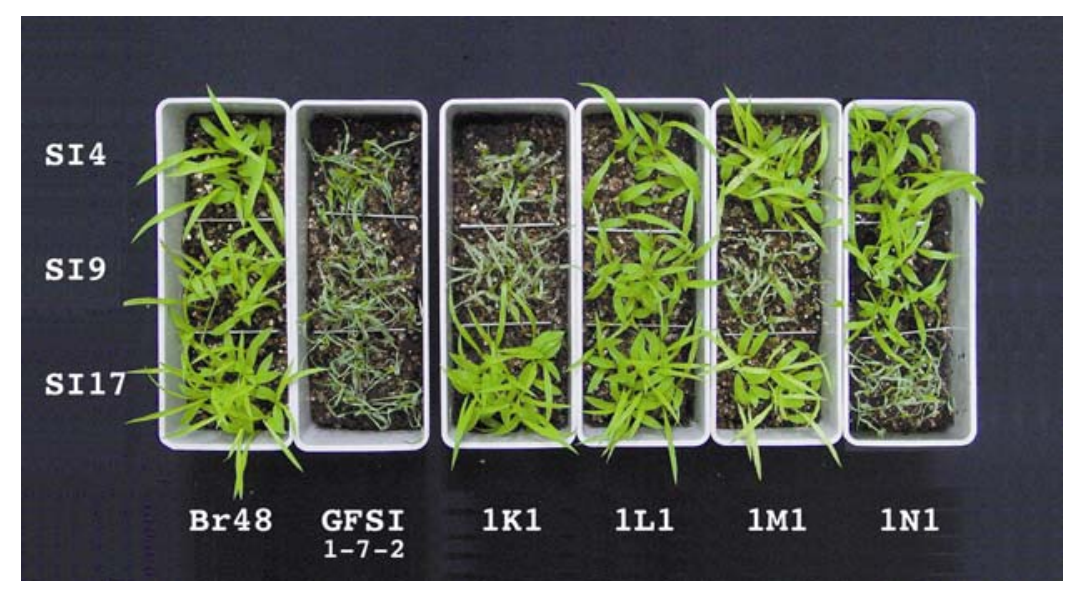

Fig. 1. Pathogenicity of Magnaporthe grisea field isolates (Br48 and GFSI1-7-2) and their $\mathrm{F}_{1}$ progeny (1K1, 1L1, 1M1, and 1N1) on foxtail millet cvs. Ki-awa (SI4), Beni-awa (SI9), and Kariwano-zairai (SI17). 1K1, 1L1, 1M1, and 1N1 are tetrads derived from a single ascus. 
$P f m l$ and, therefore, that $P f m l$ is not involved in the specificity on SI17. The segregation pattern on SI17 was also compared with that on SI4 to determine if Pfm2 is involved in the specificity on SI17. The combined segregation was not significantly different from 5:1:1:1, which is expected when one of the two loci is Pfm2, nor from 9:3:3:1, which is expected when both loci are different from Pfm2 (12).

Although dominance of alleles at the Pfml and Pfm2 loci cannot be unequivocally determined in this haploid fungus, we designate avirulence alleles from the Triticum isolate as PFM1 and $P F M 2$ and virulence alleles from the Setaria isolate as $p f m 1$ and pfm2. This is based on the fact that the segregation of avirulence to virulence was not 1:3 but 3:1 on SI4 (Table 2; Fig. 1). The results described previously can be summarized by assigning these gene symbols to the tetrads in Figure 1. 1L1 and 1N1 are avirulent on SI9 because they carry PFM1, whereas $1 \mathrm{~K} 1$ and $1 \mathrm{M} 1$ are virulent on it because they carry $p f m 1.1 \mathrm{~L} 1$ and $1 \mathrm{~N} 1$ are also avirulent on SI4 because PFM1 operates on SI4. 1M1 is avirulent on SI4 due to PFM2. On SI17, the Pfml locus should not be involved because the $P F M 1$ carrier $1 \mathrm{~N} 1$ is virulent and the $p f m 1$ carrier $1 \mathrm{M} 1$ is avirulent.

The segregation data on SI4, SI9, and SI17 were combined with previously reported data (9) on wheat cv. Norin 4 (N4). In all combinations of cultivars there occurred hybrid cultures that were virulent on both wheat and foxtail millet (data not shown). As reported previously (9), the specificity of the Setaria isolate GFSI1-7-2 and the Triticum isolate Br48 on wheat is mainly controlled by two loci, $P w t 1$ and $P w t 2$. Avirulence alleles from the Setaria isolates were designated as $P W T 1$ and $P W T 2$, and virulence alleles from the Triticum isolates were designated as pwt 1 and $p w t 2$ (11). When the segregation data of $P f m l$ were combined with those of $P w t 1$, the possible four genotypes occurred in a
1:1:1:1 ratio (Table 4), indicating that those loci are inherited independently. When $P f m l$ was combined with $P w t 2$, the possible four genotypes also occurred (Table 4), but the recombinants $(P ; Q$ and $p ; q)$ were more frequent than the parental types for unknown reasons. Such analyses were not possible for Pfm 2 because $\mathrm{F}_{1}$ cultures carrying $P F M 1$ alone and those carrying both $P F M 1$ and PFM2 showed the same phenotype (avirulent on both SI9 and SI4) and were not distinguishable.

\section{DISCUSSION}

Two loci were identified that are involved in the pathogenic specificity of the Triticum isolate and the Setaria isolate toward foxtail millet. Their specific parasitism on SI9 and SI10 was controlled by the same single locus, Pfml, whereas that on SI4 was controlled by two loci, Pfml and Pfm2. The segregation data on SI9, SI10, and SI4 were summarized in Table 5 with genotypes of the $\mathrm{F}_{1}$ cultures. Genotypes of the parental Triticum isolate ( $\left.\mathrm{Br} 48\right)$

TABLE 5. A hypothesis for genetic mechanisms of the interaction between foxtail millet cultivars and $\mathrm{F}_{1}$ cultures derived from the cross Br48 $\times$ GFSI1$7-2$

\begin{tabular}{lcc}
\hline & \multicolumn{2}{c}{ Reaction with $^{\mathrm{a}}$} \\
\cline { 2 - 3 } Genotype of $\mathrm{F}_{1}$ cultures & SI9 and SI10 $\left(R_{1} ; r_{2}\right)^{\mathrm{b}}$ & SI4 $\left(R_{1} ; R_{2}\right)$ \\
\hline$P F M 1 ; P F M 2$ & $\mathrm{~A}\left[P F M 1-R_{1}\right]^{\mathrm{c}}$ & $\mathrm{A}\left[P F M 1-R_{1}, P F M 2-R_{2}\right]$ \\
$P F M 1 ; p f m 2$ & $\mathrm{~A}\left[P F M 1-R_{1}\right]$ & $\mathrm{A}\left[P F M 1-R_{1}\right]$ \\
pfm1;PFM2 & $\mathrm{V}[-]$ & $\mathrm{A}\left[P F M 2-R_{2}\right]$ \\
pfm1;pfm2 & $\mathrm{V}[-]$ & $\mathrm{V}[-]$ \\
\hline
\end{tabular}

${ }^{\mathrm{a}} \mathrm{A}$, avirulent; $\mathrm{V}$, virulent.

${ }^{\mathrm{b}}$ Plant genotype.

${ }^{c}$ Avirulence gene-resistance gene pair involved in the reaction.

TABLE 2. Pathogenicity to foxtail millet cultivars of $F_{1}$ cultures derived from the cross $\mathrm{Br} 48 \times$ GFSI1-7-2

\begin{tabular}{|c|c|c|c|c|c|c|c|c|c|c|}
\hline \multirow[b]{2}{*}{ Cultivar } & \multicolumn{8}{|c|}{ Number of $F_{1}$ cultures } & \multicolumn{2}{|c|}{$X^{2 b}$} \\
\hline & $0-^{\mathrm{a}}$ & $1-$ & $2-$ & $3-$ & $4-5$ & Total & Avirulent (index $<3$ ) & Virulent (index $\geq 3$ ) & $1: 1$ & $3: 1$ \\
\hline SI4 & 20 & 31 & 2 & 15 & 1 & 69 & 53 & 16 & $19.84 * *$ & 0.12 \\
\hline SI9 & 11 & 23 & 2 & 18 & 15 & 69 & 36 & 33 & 0.13 & $19.17 * *$ \\
\hline SI12 & 63 & 6 & 0 & 0 & 0 & 69 & 69 & 0 & - & - \\
\hline SI17 & 18 & 28 & 7 & 13 & 3 & 69 & 53 & 16 & $19.84 * *$ & 0.12 \\
\hline
\end{tabular}

${ }^{a}$ The affected area was rated using six progressive grades from 0 to 5: 0 , no visible symptoms; 1 , diseased leaf area of up to 5\% of the whole leaf, mainly composed of brown pinpoint spots; 2 , diseased leaf area of up to $10 \%$, mainly composed of small brown lesions; 3 , diseased leaf area of up to $50 \%$, mainly composed of green lesions with an intermediate size; 4, diseased leaf area of up to 80\%, mainly composed of green large lesions; and 5, diseased leaf area of more than $80 \%$, almost completely shriveled. The mean disease severity on eight leaves was used as the virulence index (VI) of each culture. Isolates with VI less than 3 were considered avirulent, whereas those with VI greater than or equal to 3 were considered virulent.

$\mathrm{b} * *$ indicate significant at the $1 \%$ level.

TABLE 3. Combined segregation of pathogenicity to two cultivars of foxtail millet in $F_{1}$ cultures derived from the cross Br48 $\times$ GFSI1-7-2

\begin{tabular}{|c|c|c|c|c|c|c|c|c|}
\hline \multicolumn{2}{|c|}{ Cultivar } & \multicolumn{5}{|c|}{ Number of $F_{1}$ cultures } & \multicolumn{2}{|c|}{$X^{2 b}$} \\
\hline $\mathrm{p}$ & $\mathrm{q}$ & $\mathrm{ApAq}^{\mathrm{a}}$ & $\mathrm{ApVq}$ & $\mathrm{VpAq}$ & $\mathrm{VpVq}$ & Total & $2:(0): 1: 1$ & $3: 1: 3: 1$ \\
\hline SI9 & SI10 & 36 & 0 & 0 & 33 & 69 & & \\
\hline SI9 & SI4 & 36 & 0 & 17 & 16 & 69 & 0.16 & $23.76^{* * *}$ \\
\hline
\end{tabular}

${ }^{a} \mathrm{~A}$, avirulent; V, virulent. For example, ApVq represents cultures that are avirulent on $\mathrm{p}$ and virulent on $\mathrm{q}$.

$\mathrm{b} * *$ indicate significant at the $1 \%$ level.

TABLE 4. Combined segregation of $P f m 1$ and $P w t$ alleles in $F_{1}$ cultures derived from the cross Br48 $\times$ GFSI1-7-2

\begin{tabular}{|c|c|c|c|c|c|c|}
\hline \multicolumn{2}{|c|}{ Locus } & \multicolumn{5}{|c|}{ Number of $F_{1}$ cultures } \\
\hline $\mathrm{P} / \mathrm{p}$ & $\mathrm{Q} / \mathrm{q}$ & $\mathrm{P} ; \mathrm{Q}^{\mathrm{a}}$ & $\mathrm{P} ; \mathrm{q}$ & $\mathrm{p} ; \mathrm{Q}$ & $\mathrm{p} ; \mathrm{q}$ & Total \\
\hline Pfm 1 & $P w t 1$ & 18 & 18 & 19 & 14 & 69 \\
\hline Pfml & Pwt2 & 24 & 12 & 8 & 25 & 69 \\
\hline
\end{tabular}

${ }^{\mathrm{a}}$ Genotypes. The capital and small letters represent avirulence and virulence alleles, respectively. 
and Setaria isolate (GFSI1-7-2) are presumed to be PFM1;PFM2 and $p f m 1 ; p f m 2$, respectively. A cross between them should yield four genotypes in the $\mathrm{F}_{1}$ population, i.e., PFM1;PFM2 (I), PFM1;pfm2 (II), pfm1;PFM2 (III), and pfm1;pfm2 (IV). On SI9 and SI10, the Pfm2 locus has no effect on the phenotype and only the $P f m 1$ locus is involved in the specificity. So, $P F M 1$ progeny (I and II) are avirulent while $p f m 1$ progeny (III and IV) are virulent, resulting in a 1:1 segregation in the $\mathrm{F}_{1}$ population. On SI4 both loci are involved, resulting in a 3:1 segregation. Do Pfml and $P f m 2$ govern avirulence or virulence? Are functional alleles $P F M 1$ and PFM2 (avirulence alleles from the Triticum isolate) or $\mathrm{pfm} 1$ and pfm 2 (virulence alleles from the Setaria isolate)? We assume that they are both avirulence loci because of the way they interact epistatically; one copy of either gene is sufficient to cause avirulence in the $\mathrm{F}_{1}$ population (Fig. 1; Tables 2 and 5).

How do these genes govern the specificity? A clue may be in our data shown in Table 3 and Figure 1. These data indicate that Triticum and Setaria isolates carry no "master gene" that operates on all foxtail millet cultivars commonly for determining their parasitic specificity on Setaria italica. Fungal genes involved in the specificity are different from cultivar to cultivar. This variation can be explained by gene-for-gene interactions (2); the variation of fungal genes involved may be due to the variation of resistance genes carried by the cultivars. A very similar situation has been already reported in the forma specialis-genus specificity in the powdery mildew (Blumeria graminis)-cereal system. Tosa and coworkers crossed B. graminis f. sp. tritici (the wheat mildew fungus) with $B$. graminis f. sp. agropyri (the wheatgrass mildew fungus) and identified four fungal genes that are involved in their parasitic specificity on wheat $(12,13,15)$. They subsequently identified their corresponding resistance genes in wheat cultivars (15-17) and demonstrated that the forma specialis-genus specificity is governed by gene-for-gene interactions (13-15). The fungal genes were all avirulence genes from the wheatgrass mildew fungus that are involved in its specificity on the genus Triticum. However, they were cultivar-specific in their effects and operated in various combinations on each wheat cultivar (15). This cultivarspecific operation of the avirulence genes could be attributed to the difference of resistance genes carried by each cultivar (15). The specificity between foxtail millet cultivars and Setaria/Triticum isolates of $M$. grisea can be conditioned by a similar genetic mechanism and may be an example that species specificity is governed by gene-for-gene interactions.

With this hypothesis, the interactions between the $F_{1}$ cultures and the foxtail millet cultivars are explained as follows (Table 5). Resistance genes corresponding to PFM1 and PFM2 are tentatively designated as $R 1$ and $R 2$, respectively. On SI4 both PFM1 and $P F M 2$ are involved in the specificity because this cultivar carries both $R 1$ and $R 2$. On SI9 PFMI is involved but PFM2 is not, because this cultivar carries $R 1$ but not $R 2$.

Kato (6) found cultivar specificity between three Setaria isolates and 20 foxtail millet cultivars. The resistance of SI12 to GFSI1-7-2 reported in the present study may be an example of such specificity; SI12 may carry a resistance gene and GFSI1-7-2 may carry its corresponding avirulence gene. It should be noted that the cross between Br48 and GFSI1-7-2 did not produce progeny that is virulent on SI12. A simple interpretation is that $\mathrm{Br} 48$ also carries the avirulence gene corresponding to the resistance gene in SI12. This is comparable to the fact that the rye mildew fungus, B. graminis f. sp. secalis, carries avirulence genes corresponding to wheat genes for resistance to races of the wheat mildew fungus, B. graminis f. sp. tritici (8).

\section{ACKNOWLEDGMENTS}

This work was supported by grants from the Ministry of Education, Culture, Sports, Science and Technology, Japan (10460022). We thank H. Kato, the former professor of Kobe University, for valuable suggestions and comments.

\section{LITERATURE CITED}

1. Day, P. R. 1976. Gene functions in host-parasite systems. Pages 65-73 in: Specificity in Plant Disease. R. K. S. Wood and A. Graniti, eds. Plenum Press, New York.

2. Flor, H. H. 1956. The complementary genetic systems in flax and flax rust. Adv. Genet. 8:29-54.

3. Heath, M. C. 1981. A generalized concept of host-parasite specificity. Phytopathology 71:1121-1123.

4. Heath, M. C. 1991. The role of gene-for-gene interactions in the determination of host species specificity. Phytopathology 81:127-130.

5. Kang, S., Sweigard, J. A., and Valent, B. 1995. The $P W L$ host specificity gene family in the blast fungus Magnaporthe grisea. Mol. Plant-Microbe Interact. 8:939-948.

6. Kato, H. 1983. Responses of Italian millet, oat, timothy, Italian ryegrass and perennial ryegrass to Pyricularia species isolated from cereals and grasses. (In Japanese) Proc. Kanto-Tosan Plant Prot. Soc. 30:22-23.

7. Kato, H., Yamamoto, M., Yamaguchi-Ozaki, T., Kadouchi, H., Iwamoto, Y., Nakayashiki, H., Tosa, Y., Mayama, S., and Mori, N. 2000. Pathogenicity, mating ability and DNA restriction fragment length polymorphisms of Pyricularia populations isolated from Gramineae, Bambusideae and Zingiberaceae plants. J. Gen. Plant Pathol. 66:30-47.

8. Matsumura, K., and Tosa, Y. 1995. The rye mildew fungus carries avirulence genes corresponding to wheat genes for resistance to races of the wheat mildew fungus. Phytopathology 85:753-756.

9. Murakami, J., Tosa, Y., Kataoka, T., Tomita, R., Kawasaki, J., Chuma, I., Sesumi, Y., Kusaba, M., Nakayashiki, H., and Mayama, S. 2000. Analysis of host species specificity of Magnaporthe grisea toward wheat using a genetic cross between isolates from wheat and foxtail millet. Phytopathology 90:1060-1067.

10. Sweigard, J. A., Carroll, A. M., Kang, S., Farrall, L., Chumley, F. G., and Valent, B. 1995. Identification, cloning, and characterization of PWL2, a gene for host species specificity in the rice blast fungus. Plant Cell 7:1221-1233.

11. Takabayashi, N., Tosa, Y., Oh, H. S., and Mayama, S. 2002. A gene-forgene relationship underlying the species-specific parasitism of Avenal Triticum isolates of Magnaporthe grisea on wheat cultivars. Phytopathology 92:1182-1188.

12. Tosa, Y. 1989. Genetic analysis of the avirulence of wheatgrass powdery mildew fungus on common wheat. Genome 32:913-917.

13. Tosa, Y. 1989. Evidence on wheat for gene-for-gene relationship between formae speciales of Erysiphe graminis and genera of gramineous plants. Genome 32:918-924.

14. Tosa, Y. 1994. Gene-for-gene interactions between the rye mildew fungus and wheat cultivars. Genome 37:758-762.

15. Tosa, Y., and Sakai, K. 1990. The genetics of resistance of hexaploid wheat to the wheatgrass powdery mildew fungus. Genome 33:225230.

16. Tosa, Y., Tokunaga, H., and Ogura, H. 1988. Identification of a gene for resistance to wheatgrass powdery mildew fungus in the common wheat cultivar Chinese Spring. Genome 30:612-614.

17. Tosa, Y., Tsujimoto, H., and Ogura, H. 1987. A gene involved in the resistance of wheat to wheatgrass powdery mildew fungus. Genome 29:850-852.

18. Urashima, A. S., Hashimoto, Y., Don, L. D., Kusaba, M., Tosa, Y., Nakayashiki, H., and Mayama, S. 1999. Molecular analysis of the wheat blast population in Brazil with a homolog of retrotransposon MGR583. Ann. Phytopathol. Soc. Jpn. 65:429-436.

19. Urashima, A. S., Igarashi, S., and Kato, H. 1993. Host range, mating type and fertility of Pyricularia grisea from wheat in Brazil. Plant Dis. 77:1211-1216.

20. Yaegashi, H. 1978. Inheritance of pathogenicity in crosses of Pyricularia isolates from weeping lovegrass and finger millet. Ann. Phytopathol. Soc. Jpn. 44:626-632. 